\section{IDENTIFICATION OF SHARED TUMOR EPITOPES FROM ENDOGENOUS RETROVIRUSES INDUCING HIGH AVIDITY CYTOTOXIC T CELLS FOR CANCER IMMUNOTHERAPY}

${ }^{1}$ Paola Bonaventura*, ${ }^{2}$ Vincent Alcazer, ${ }^{1}$ Virginie Mutez, ${ }^{3}$ Laurie Tonon, ${ }^{4}$ Juliette Martin, ${ }^{1}$ Nicolas Chuvin, ${ }^{1}$ Rasha Boulos, ${ }^{1}$ Yann Estornes, ${ }^{2} J$ enny Valladeau-Guilemond, ${ }^{3}$ Alain Viari, ${ }^{2}$ Christophe Caux, ${ }^{1}$ Stephane Depil. 'ErVaccine technologies, Lyon, France; ${ }^{2}$ Centre de Recherche en Cancerologie Lyon, Lyon, France; ${ }^{3}$ Synergie Lyon Cancer, Lyon, France; ${ }^{4}$ CNRS, Lyon, France

Background Human endogenous retroviruses (HERVs) are aberrantly expressed by tumor cells and may represent a source of $\mathrm{T}$ cell epitopes

Methods Using TCGA pancancer RNAseq data $(\mathrm{n}=8,893$ samples), we developed a bioinformatics-based method to select cancer-specific HERVs associated with a cytotoxic $T$ cell response ("cyt-HERVs") and identify shared $T$ cell epitope candidates. $T$ cells were primed with selected short and long peptide candidates from HLA-A2+ healthy donors. Peptidespecific dextramers were used to sort and expand specific $\mathrm{CD} 8+\mathrm{T}$ cell clones and determine their TCR sequences and avidity. Cytotoxicity was assessed against HERV-expressing tumor cell lines and patient-derived organoids using Incucyte and Nanolive technologies (Flowchart, figure 1).

Results In a pancancer analysis, we identified 57 HML-2/ HERV-K HLA-A*0201 epitope candidates from 27 distinct open reading frames. Six shared HLA-A2 strong binders 9-mer peptides, present on multiple HERVs located on different chromosomes, and with translational evidence found in mass spectrometry public datasets, were selected and synthetized. In vitro HLA binding assay confirmed peptide-HLA affinity. Priming assays showed the presence of specific CD8 $+\mathrm{T}$ cells leading to polyfunctional IFN- $\gamma+$ TNF- $\alpha+$ T cell responses with upregulation of the degranulation marker CD107A upon co-culture with peptide-pulsed T2 cells. Synthetic long peptides containing the epitopes were used to confirm the correct processing by antigen-presenting cells. The functionality of the sorted $\mathrm{T}$ cell clones was confirmed using an Elispot assay $(\mathrm{GrzB}+\mathrm{IFN}-\gamma+)$. Their sequenced TCRs were predicted to stably interact with their respective MHC-peptide complexes in a 3D model. This was confirmed by measurement of the functional avidity, which was in the same order as CMV-specific $\mathrm{T}$ cell clones. HERV-specific CD $8+\mathrm{T}$ cells induced specific cell death of HLA-A2+ cancer cell lines, associated with IFN-g production, in a HLA-A2 restricted manner. Finally, pre-existing HERV-specific CD8 + T cells were identified using dextramers among tumor infiltrating lymphocytes (TILs) from cancer patients. HERV-specific T cells co-cultured with patient derived organoids showed signs of activation with lysis of the organoid.

Conclusions Our bioinformatics-based approach allowed us to identify shared HERV-derived CD8 $+\mathrm{T}$ cell epitopes specifically expressed by tumor cells and inducing high avidity $\mathrm{T}$ cell clones able to kill tumor cells in a class I-restricted manner. The detection of TILs recognizing HERV peptides suggests natural presentation of these epitopes in the tumors. These HERV-derived epitopes may thus represent relevant targets for the development of new cancer vaccines or T cell-based therapies, especially in tumors with low mutational burden.

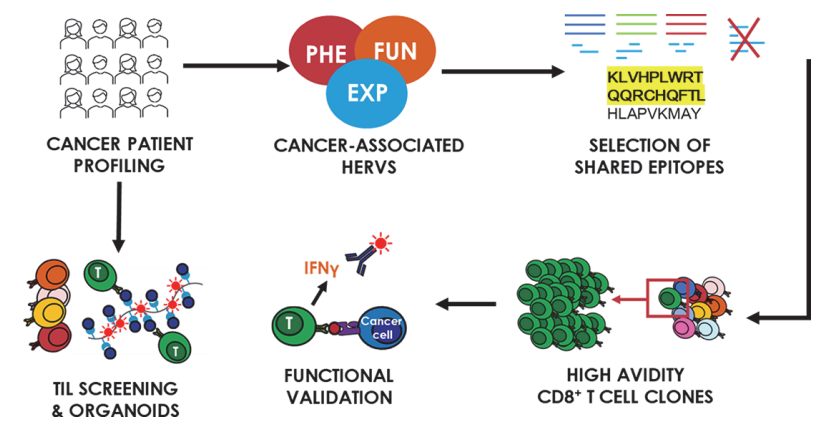

Abstract 691 Figure 1 Graphical flowchart of HERV antigen validation. Graphical representation of the flowchart used to identify and validate specific CD8+ T cells for shared tumor epitopes from endogenous retroviruses http://dx.doi.org/10.1136/jitc-2021SITC2021.691

http://dx.doi.org/10.1136/jitc-2021-SITC2021.691 